\title{
Can Teenagers Control a 3D Racing Game using Motion-onset Visual Evoked Potentials?
}

Beveridge, R., Wilson, S., \& Coyle, D. (2017). Can Teenagers Control a 3D Racing Game using Motion-onset Visual Evoked Potentials? Brain-Computer Interfaces, 4(1-2:SI), 102-113.

https://doi.org/10.1080/2326263X.2016.1266725

Link to publication record in Ulster University Research Portal

\section{Published in:}

Brain-Computer Interfaces

Publication Status:

Published (in print/issue): 01/01/2017

DOI:

10.1080/2326263X.2016.1266725

\section{Document Version}

Author Accepted version

\section{General rights}

Copyright for the publications made accessible via Ulster University's Research Portal is retained by the author(s) and / or other copyright owners and it is a condition of accessing these publications that users recognise and abide by the legal requirements associated with these rights.

\section{Take down policy}

The Research Portal is Ulster University's institutional repository that provides access to Ulster's research outputs. Every effort has been made to ensure that content in the Research Portal does not infringe any person's rights, or applicable UK laws. If you discover content in the Research Portal that you believe breaches copyright or violates any law, please contact pure-support@ulster.ac.uk. 


\title{
Can Teenagers Control a 3D Racing Game using Motion-onset Visual Evoked Potentials?
}

\author{
Ryan Beveridge, Shane Wilson and Damien Coyle \\ Intelligent Systems Research Centre, Ulster University, Londonderry, Northern Ireland, U.K. \\ Beveridge-R@email.ulster.ac.uk, s.wilson@ulster.ac.uk and dh.covle@ulster.ac.uk
}

\begin{abstract}
Motion-onset visual evoked potentials (mVEPs) are time and phase-locked brain responses to motion-related stimuli. An mVEP response provides robust features for brain-computer interface (BCI) applications and have the added benefit of being less visually fatiguing than other visual evoked potentials (VEPs). In this study an mVEP BCI that enables control of a visually rich, 3-dimensional (3D) car-racing video-game is evaluated. A group of fifteen teenage school children (13-16 years old) participated in a single session while they attended a summer school. Participants were asked to control the direction of a car within a realistic racing circuit, where the position of the car was controlled by focusing on one of five motion-related stimuli. Classification accuracy (\%) and information transfer rate (ITR) (bits per minute (bpm)) results were encouraging, with participants achieving an average online accuracy of $72 \%(12 \mathrm{bpm})$ in the first lap, $67 \%$ (10bpm) in the second lap and 65\% (10bpm) in the third lap (chance accuracy and ITR is $20 \%$ and zero bpm). The study shows for the first time the feasibility of using the mVEP paradigm in a commercial-grade car-racing video-game. It is also one of the first reports on the performance of a group of teenagers using a BCI.
\end{abstract}

Keywords - brain-computer interface (BCI); motion-onset visually evoked potentials (mVEP); electroencephalography (EEG); video game; 3-dimensional (3D).

\section{Introduction}

A brain-computer interface (BCI) system allows musclefree control over a computerised application [1]. Traditionally, the target end-user group for BCIs has been the physically impaired, with studies investigating, e.g., spelling applications for communication needs [2][3][4], personal transport and prosthesis control [5][6] and BCIbased rehabilitation applications [7][8]. With the ubiquitous nature of powerful and inexpensive computing technology in recent years and the extensive investment and effort in developing more advanced signal processing strategies [9][10][11], the application domain for BCI use has broadened significantly, now including video gamers and keen interest from the video games industry [12][13][14]. In an industry worth almost $\$ 100$ billion worldwide, the video games industry is a steadily growing market [15] and represents an ideal demographic audience for BCI technology where the typical user may appreciate the novelty and learning challenges faced by novel interaction techniques and control modalities such as a brain-computer games interaction (BCGI) [14].

Visual evoked potentials (VEPs) form the subset of BCI paradigms which utilise visual stimuli to evoke responses in the cortical activity of a BCI user. Typically, a number of stimuli are presented to the user either on a computer screen or via lighting panels - each representing a different command for the BCI system to process and execute. In the case of electroencephalography (EEG), as used in this study to read cortical activity, these responses can be detected in the ongoing EEG in real-time.
Popular VEPs for use in BCIs include the $\mathrm{P} 300$ potential [2], a positive voltage in the recorded EEG occurring at around 250-500 milliseconds (ms) following the presentation of a rare stimulus among frequently presented stimuli, known as the "oddball paradigm" [16]. P300 potentials have been successfully used in BCI spelling applications [2][17][18][19] and BCI video games [20][21][22]. Steady-state VEPs (SSVEP) are a type of VEP which employ constantly flashing or flickering stimuli at particular frequencies. The constant flashing enters the brain into a "steady-state" of cortical activity and the effect is observed in the EEG as a waveform with a frequency matching the frequency of the flashing or flickering stimulus and its harmonics. SSVEP has been successfully used in many BCI applications including BCI spellers [23][24]. Chen et al. [4] employ SSVEP for a BCI speller and report the highest information transfer rates (ITR) of any BCI to date $(5.32 \mathrm{bits} / \mathrm{sec})$, achieved by using the joint frequency phase modulation (JFPM) method to enhance discriminability between stimuli of narrow frequency ranges. SSVEP has also been successfully used for wheelchair control [5], prosthesis control [6] and BCI gaming [25][26]. Code-modulated VEP (cVEP) also use flashing imagery to evoke a response in the EEG and work by flashing stimuli with particular code-modulated sequences. cVEP is a relatively new BCI paradigm and studies so far have concentrated around communication applications as in BCI spellers [27], computer input devices [28] and for control in a virtual environment [29]. Although the above-mentioned types of VEP produce robust and reliable communication rates between the user and 
computer, without the need for long user training sessions, they share a common caveat i.e., due to the reliance on flashing stimuli, they can cause visual fatigue for the userparticularly after a period of continuous use.

Motion-onset VEPs (mVEPs) use motion-related stimuli to evoke a response in the EEG [30][3]. An mVEP occurs in the EEG response after the sudden motion of a moving stimulus. The perception of motion occurs in the primary visual cortex and extends to the medial temporal (MT) and medial superior temporal (MST) areas of the visual cortex [31][32].

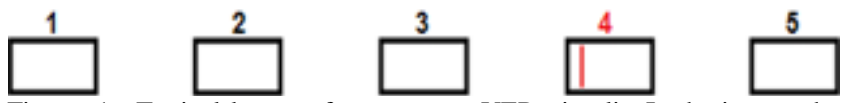

Figure. 1. Typical layout of on-screen mVEP stimuli. In the image, the number 4 stimuli is the current target (to which the user should attend visually) and is also the currently active stimuli (i.e., the red line is in motion and moving from right to left).

To evoke a response for motion, the user gazes at their required stimuli which are delineated on a computer screen (Fig. 1). Typically, stimuli comprise a black-coloured rectangle shape $1.24^{\circ}$ in width and $0.76^{\circ}$ in height with a normally vacant plain white centre. A red-coloured line $0.66^{\circ}$ in height moves from the right hand side to the left hand side of the white centre in a quick horizontal motion (Fig. 2).

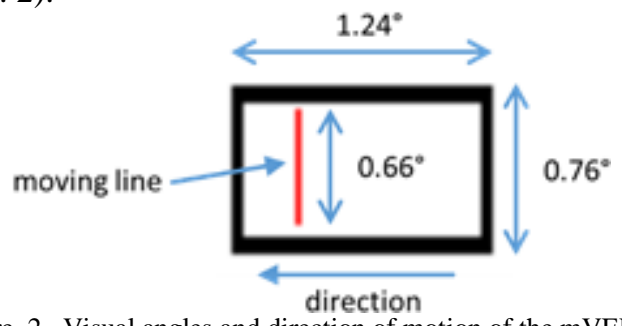

Figure. 2. Visual angles and direction of motion of the mVEP stimuli.

As a consequence of the motion-related rather than flashing stimuli, an mVEP-based BCI paradigm presents a more elegant and less visually fatiguing BCI paradigm than P300, SSVEP or cVEP. mVEP responses are time and phase-locked to the onset of motion and consist of three main components, separable in time and positivity [33] (fig. 3 ). The P100 positive peak occurs at around $100 \mathrm{~ms}$ poststimulus and its early phase (80-110ms) emanates from the lateral extrastriate cortex and the later phase $(110-140 \mathrm{~ms})$ emanates from the ventral occipito-temporal cortex [34]. The P100 is immediately followed by the motion-specific negative N200 peak at around 160-200ms. N200 is the most prominent component and is generated from the extrastriate temporo-occipital and associate parietal areas [35]. Finally, the P200, whose amplitude can be increased with more complex moving stimuli, has a latency of between 240$500 \mathrm{~ms}$ and emanates from parietal up to central areas.



Figure. 3. The three main features of the mVEP response.

mVEPs were first investigated in BCI in [30] where an mVEP-based BCI control scheme was implemented to investigate the users' responses to moving stimuli. In [36] mVEP stimuli were employed as a control modality for three online BCI video games of different genres and in [37], a follow-up study tested the same three BCI games using a heads-up display (HUD) in which the stimuli were presented on a white background as opposed to overlaying mVEP stimuli onto the games graphics. The results indicated a HUD with white background would likely enable better mVEP detection accuracy in such games. In [38] mVEP stimuli were employed as a control modality to manoeuvre a humanoid robot in real-time.

In order to progress the field of BCI controlled video gaming or neuro-gaming, it is important to consider the consequences of VEP stimuli employed within visually-rich video games with fast-paced, high-fidelity and brightly coloured graphics. In a previous study [39] we investigated the mVEP paradigm within a BCI video game environment using five different levels of graphical complexity where five mVEP evoking stimuli were presented. We found evidence to suggest that as the graphical complexity increased, the accuracy of detecting mVEPs decreased. In a second study, to investigate the effects on mVEP accuracy using popular, commercially available video games from various genres and graphical complexities [40], we employed five commercially available video games with five $\mathrm{mVEP}$ stimuli presented simultaneously with the video games. Our findings indicated that graphical complexity alone does not significantly degrade mVEP accuracies, whilst some of the more primitive properties of video games, such as the use of primary colours and pace, do have an effect on the mVEP detection accuracy. Also, results from the study showed that the car racing game Gran Turismo 3 (2001) [41], previously available on the Sony Playstation 2 games console [42], consistently produced the greatest accuracies across subjects. These findings verified that the uniformly paced gameplay and realistic colour palette used within the Gran Turismo 3 game level provided a gaming environment that minimises the impact on mVEP detection and similar games would likely provide maximum mVEP control accuracy, whereas fast-paced games such as Crash Bandicoot [43] had more of an impact on the reliability of detecting mVEPs from EEG. In a further study [44], we used an Oculus Rift [45] virtual-reality (VR) headset and investigated the effects on mVEP accuracy while users were subjected to two different levels of graphical complexity. Our findings showed the feasibility of employing a VR device as a display modality for an $\mathrm{mVEP}$ game 
environment with no discernible difference between mVEP detection accuracy in VR and standard desktop computer monitor display.

Following on from the findings of [40], in the current study, we employ the mVEP paradigm as a control modality for an EEG-based BCI-controlled video game. We employ a real-time controlled, custom-made 3D car-racing videogame and investigate the performance of fifteen teenagers playing the game in a single session.

\section{Methodology}

\subsection{Data Acquisition Setup}

Fifteen BCI naïve teenage participants, four female and 11 male, of high-school age (age range 13-16 years) took part in the study and were recruited from a group participating in a summer school at Ulster University. Ethical approval was granted by the Ulster University Research Ethics Committee (UREC) and written parental informed consent was granted by parents of the children and written assent by the children. All participants had normal or corrected to normal hearing and vision and had no other health related conditions that breached inclusion criteria for the study. Each participant partook in a single session lasting approximately one hour. Data was recorded within an electrostatic and electromagnetic interference shielded and acoustically insulated room. Participants were seated in a fixed position on a comfortable chair in front of a $56 \mathrm{~cm}$ LCD computer monitor which presented the visual stimuli and game (see section 2.2 and 2.3 for details).

EEG data was recorded using g.tec research-grade hardware [46]. Twelve g.LADYbird active electrodes were located over visual processing areas at occipital positions $\mathrm{Cz}, \mathrm{TP} 7, \mathrm{CPz}, \mathrm{TP} 8, \mathrm{P} 7, \mathrm{P} 3, \mathrm{Pz}, \mathrm{P} 4, \mathrm{P} 8, \mathrm{O} 1, \mathrm{Oz}$ and $\mathrm{O} 2$ according to the international 10-20 system of electrode placement (Fig. 4). EEG electrodes were connected to a g.GAMMAcap electrode positioning cap and signals were amplified using a g.BSamp signal amplifier, through a g.GAMMAbox for active sensing. The raw EEG signals were digitised using a National Instruments NI6390 analogue-to-digital data acquisition card [47].

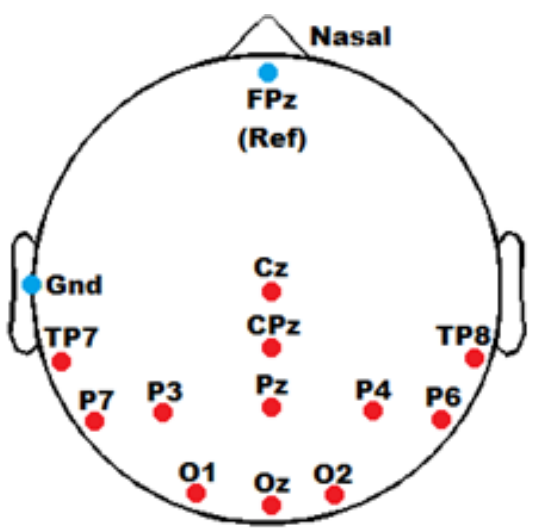

Figure. 4. The 12-channel EEG electrode montage utilised. Twelve electrodes were placed around the occipital areas according to the international 10/20 system. FPz was used as reference electrode while the left mastoid acted as ground.

To record, process and store the raw EEG signals, Matlab [48] was used. Unity 3D [49] was used to provide the visual stimulus and game environment. As Unity presented each visual stimulus, timing information was sent to a Matlab session-based interface via user datagram protocol (UDP) messages. Upon data processing of the users' chosen stimulus, Matlab returns a data value between 1 and 5 via a UDP message corresponding to the chosen stimuli back to the game running in Unity which subsequently gets processed into an in-game command.

\subsection{BCI Calibration}

At the beginning of each session, data was acquired to calibrate the BCI. Each participant was instructed to focus on stimuli overlaid onto the game environment directed by clear cues. These were presented within the same game environment in which they would play the online game, with the elimination of the car model which feeds back the detected selection to the participant during gameplay and some online specific game elements i.e., the users response was not translated into game commands during the calibration lap. A lap is defined as one complete circuit of the racing track from start to finish (Fig. 7). During the calibration lap, each of the five mVEP stimuli were activated 60 times yielding data from 300 trials. One single trial lasted for $1000 \mathrm{~ms}$ and consisted of one complete activation of each of the five stimuli in random order. During a trial, each stimuli was active for $140 \mathrm{~ms}$, followed by a pause between activation of the next randomly selected stimuli of $60 \mathrm{~ms}$. Therefore the stimulus-onset asynchrony (SOA) between stimuli activations is $200 \mathrm{~ms}$. The inter-trial interval (ITI) between two consecutive trials was $600 \mathrm{~ms}$ (Fig. 5). During the calibration lap, a visual indicator, a redcoloured on-screen arrow placed immediately above the stimuli, indicated which stimulus the participant was required to attend to i.e., the target stimulus (Fig. 6).

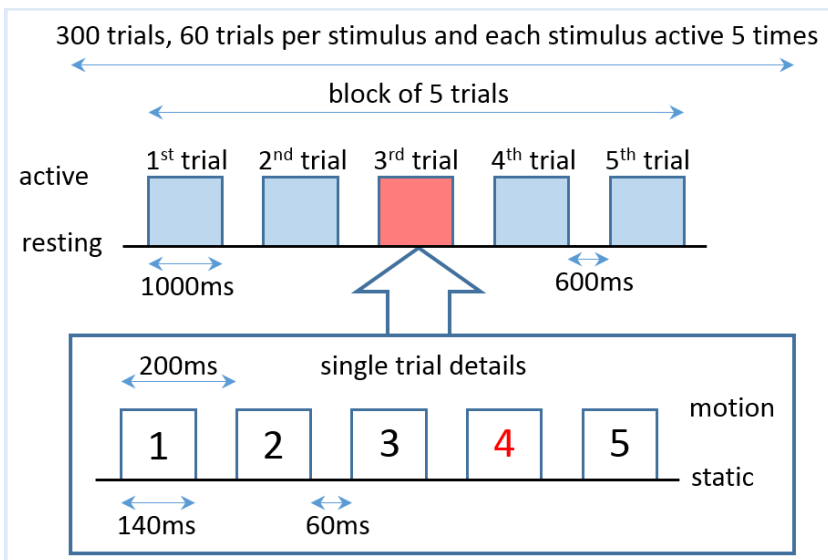

Figure. 5. Trial timing details of the calibration lap. A total of 300 trials were recorded. Each trial lasted $1000 \mathrm{~ms}$ with an ITI of $600 \mathrm{~ms}$. During a trial, each stimuli was active for $140 \mathrm{~ms}$ with a pause of $60 \mathrm{~ms}$ before onset of the next randomly selected stimulus, yielding a SOA of $200 \mathrm{~ms}$.

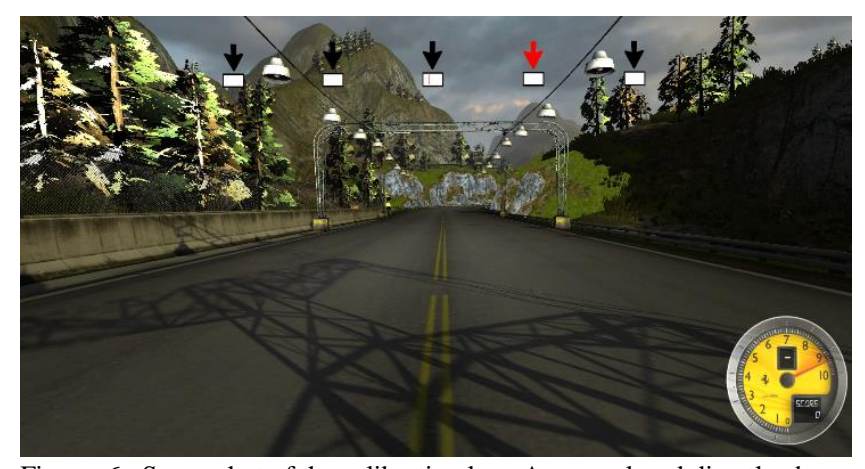

Figure. 6. Screenshot of the calibration lap. Arrows placed directly above stimuli directed the user to the target stimulus. In the example shown, stimuli four (currently indicated by a red arrow) is the current target 
stimulus and stimulus three is currently active (red moving vertical bar at a point during motion from right to left).

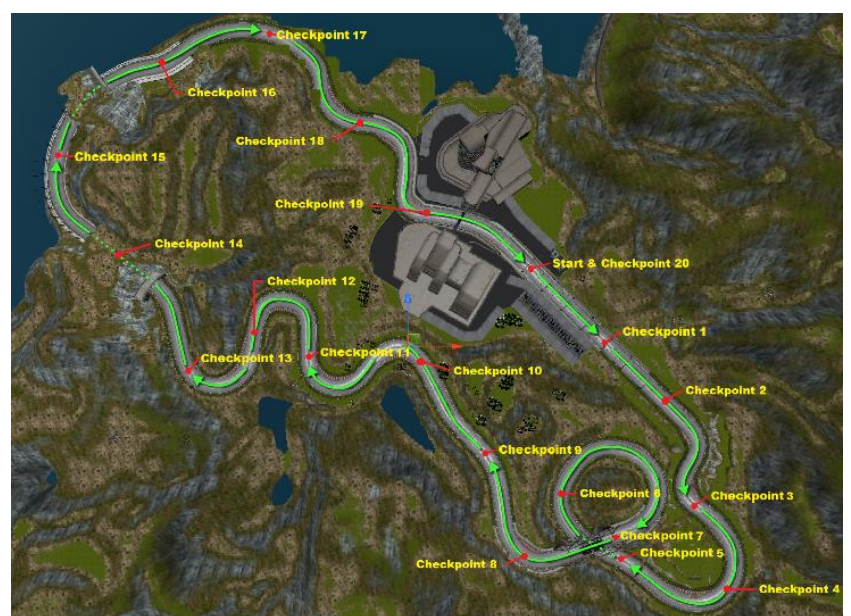

Figure. 7. An aerial view of the racing track. A total of 20 checkpoints were presented. Each participant selected a lane (stimuli were active) prior to traversing each checkpoint. During each session, three separate laps of the racing course were completed.

\subsection{Online Game Paradigm}

Using the data collected from the calibration run, an online classifier was trained (see section 3.2 for details), enabling the participant to play the game online with realtime feedback. During the online feedback, five repetitions of the stimuli occurred and the mVEP response averaged, before classification occurred.

To provide an engaging game environment and online feedback, a custom-made 3D car racing game was developed using the Unity 3D games development engine. Each participant took part in three laps of the online racing game (except participant 12 who did not complete a third lap due to a health issue). Each lap contained a total of twenty checkpoints (Fig. 7), at which the user had to control the direction of the car. The game environment included realistic graphics as found in commercially available car racing games including realistic textures, $3 \mathrm{D}$ car model, race course, racing track, speedometer (depicting the users' current score and target information), sky, mountains and foliage. The forward motion of the car was automatically controlled. The five mVEP stimuli were delineated at the top of the screen. Arrows moving in cascading motion were placed on the road surface ahead of the car in the peripheral vison of the user. These arrows directed the user to the target stimuli which they should attend to control direction/ lane position of the car at the next checkpoint (Fig. 8). Of the five arrows, four were red-coloured and one was greencoloured. All four red-coloured arrows were non-target and the user ignored. The green-coloured arrow indicated the target stimulus on which the user should focus. The position of the arrow corresponded to the on-screen position of the stimuli.

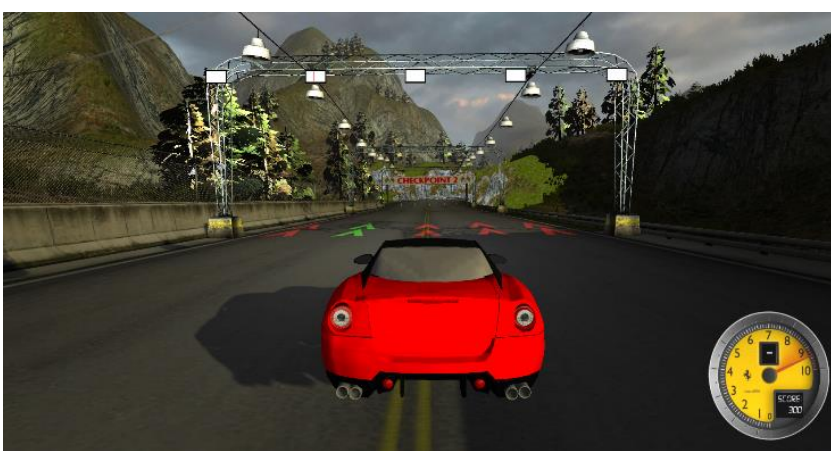

Figure. 8. Screenshot of the online level. Green-coloured arrows moving in cascading fashion directed the user to which stimuli to attend. In the image, stimuli 2 is the current target and is also currently active.

The task of the user was to select the correct racing line out of five available at the upcoming checkpoint. If the user correctly selected the target lane by focusing on the motion-related stimulus associated with the target lane, the car moved to the correct lane and a speed boost was gained whilst the car traversed the green arrow at the next checkpoint. If one of the four non-target lanes is selected, the car traverses one of the four traffic cones at the next checkpoint at a slower pace (Fig. 9). The time taken for the car to traverse the checkpoint after the users' choice is determined was based on the correct (1 second) or incorrect (5 seconds) lane choice. As each lap contained twenty checkpoints, the more correct lanes chosen by the user, the quicker they were able to complete the lap. As a further measure of success and feedback to the participant there was a scoring system. Points were awarded based on the locality of the users' chosen lane to the target lane.

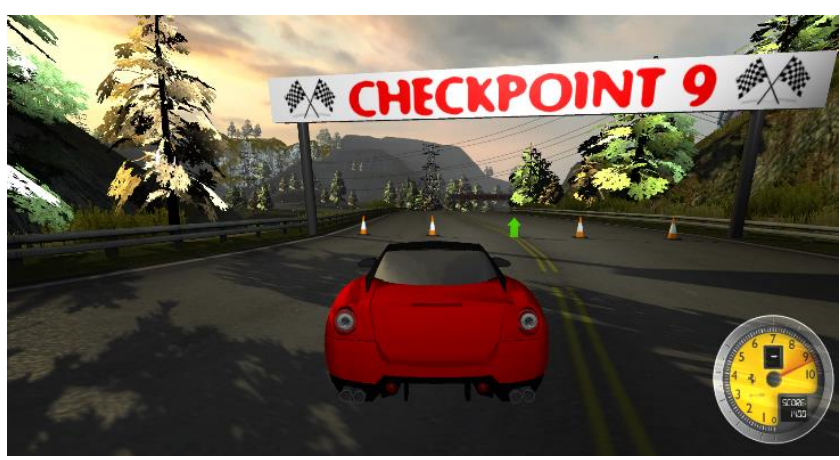

Figure. 9. Screenshot of an online lap while traversing checkpoint 9. In the image, the user selected the incorrect lane (lane 2 instead of lane 3) so traverses the second traffic cone taking 5 seconds of time.

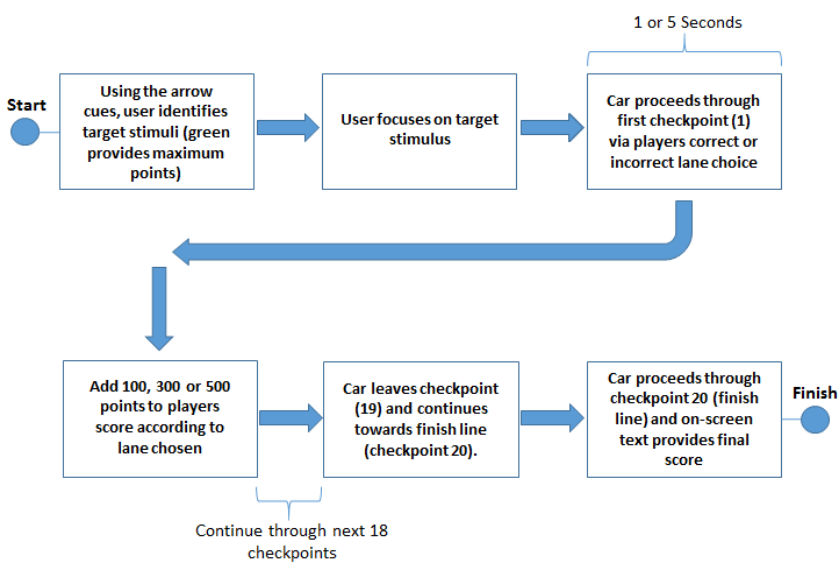

Figure. 10. The games decision-making process and lap timing details. 
If the user successfully selects the correct lane, a score of 500 points is added to the score as they traversed the checkpoint. If the user selected either of the two incorrect lanes that were closest to the correct lane a medium score of 300 was added to their points. If the user selected one of the two lanes furthest from the correct lane, a lower score of 100 points was awarded. The points system therefore penalised for incorrect lane choices but had no negative feedback and provided incentive to do well in future checkpoints. The maximum score available was 10000 points and could be achieved when the participant selected each of the correct lanes at all twenty checkpoints (i.e., 500 points $\times 20$ checkpoints $=10000$ points). The players' score therefore is indirectly related to accuracy since the player could gain three different scores i.e., 500 for correct lane choice, 300 for two closest lanes and 100 for the two furthest lanes to the target lane. Fig. 10 depicts the game process.

\section{Data Analysis}

\subsection{Data Pre-processing and Feature Extraction}

EEG was epoched with each motion onset stimulus, beginning $200 \mathrm{~ms}$ prior to the motion onset and lasting for $1200 \mathrm{~ms}$. All single trials were baseline corrected with respect to the mean voltage over the $200 \mathrm{~ms}$ preceding motion onset. EEG data was recorded using a sampling rate of $250 \mathrm{~Hz}$, digitally filtered using a low-pass Butterworth filter (order 5, with cut-off at $10 \mathrm{~Hz}$ ) and subsequently resampled at $20 \mathrm{~Hz}$. Features were extracted between the $100 \mathrm{~ms}$ and $500 \mathrm{~ms}$ epoch post-stimulus, which normally contains the most reactive $\mathrm{mVEP}$ components e.g., P100, N200 and P300. This yields nine features for each channel. Data were averaged over five trials yielding twelve feature vectors per target stimulus per lap of the game played. $\mathrm{mVEP}$ is time and phase-locked to the motion-onset stimulus, therefore, mVEP induced from the motion stimuli could be obtained through the above simple averaging procedure [30].

\subsection{Offline mVEP Classification - Training Data}

A classifier was trained using $100 \%$ (all 300 trials) of testing data gained from the calibration session. Distinguishing between target vs. non-target stimuli from each EEG channel (2-class accuracy) was the first objective. A linear discriminant analysis (LDA) classifier was trained to achieve this. A leave-p-out cross validation (LpOCV) (leave 2 out in our case - for each of the target and non-target classes) was applied to the calibration data. For each of the twelve EEG channels, LpOCV was applied and based on their mean LpOCV accuracy, each channel was ranked. The top three ranked channels were concatenated to form a new feature vector (27 features per vector) and a further LpO cross-validation was performed. Results were reported as "train - LpOCV target vs. non-target".

As there are four non-target stimuli for each target stimuli, there are number of options for selecting the nontarget stimuli trials to form the second class for training a classifier: 1) use all the data but apply a suitable classifier and performance metric for unbalanced datasets i.e., different class sizes 2) downsample the non-target stimuli data samples by randomly selecting from non-target data an equivalent number of trials and 3) upsample the target stimulus trials by copying data to balance the target class dataset size with the non-target dataset size. In previous studies the random sampling approach was applied (option 2) however this often resulted in variation in the results due to the random selection and variation in the non-target stimuli responses. To overcome this, averaging over multiple runs of the analysis with different random sampling could be applied, however, a better, less computationally intensive and more stable alternative is to use option 3 i.e., upsampling (or over sampling) e-the non-target class data by repetition of target samples. This balances the classes, ensures sufficient data for classifier training, negates randomness and maximises training accuracy and generalisation performance. This is only done on the training data.

To classify individual symbols in a single trial test i.e., 5-class discrimination, each feature vector associated with each stimulus in a trial is classified as either target or non-target. The LDA classifier produced a distance value, $D$, reflecting the distance from the hyperplane separating target and non-target features $(D>0$ for target and $D<0$ for non-target). The vector that produces the maximum distance value is selected as the classified stimulus (in some cases non-target data produces a $D>0$, however the value of $D$ is normally maximal among the target stimulus i.e., the stimulus on which the user is focused). Single trial results for five class discrimination are validated on the training data and reported as "train -validation 5-class".

\subsection{Online Game Control}

Using a classifier trained from the training data, data from the three best-ranked channels and a Matlab session-based interface for real-time processing, participants were able to play the racing game online with real-time control and feedback. During the online control, participants wait until the mVEP stimuli are presented. Each of the stimuli are presented five times (in random sequence) as the car approaches each checkpoint, allowing for a lane decision to be made for each of the twenty checkpoints i.e., the BCI makes a decision based on the mVEPs averaged over five repetitions.

\subsection{Information Transfer Rate}

To measure performance in the online laps, as well as reporting classification accuracy, we also calculate the ITR as defined in [50][51]. There are twenty checkpoints per lap and each checkpoint requires five seconds for participants to make a lane choice, yielding a time of 100 seconds of communication time per lap (i.e., 20 checkpoints $\times 5$ seconds decision time $=100$ seconds). Taking into account the $5 \mathrm{~s}$ required for each lane choice (averaging stimuli over 5 repetitions), yields 12 commands per minute. Therefore, ITR is calculated as follows:-

$b p m=\left(\log _{2}(N)+P \log _{2}(P)+(1-P) \log _{2}\left(\frac{1-P}{N-1}\right)\right) \times 12$

where $N$ is the number of classes and $P$ is the probability of correct classification. ITR is reported in bits per minute (bpm).

\section{Results}

\subsection{Offline Training Session}

Table 1 shows the offline results achieved during the calibration lap for all participants. Participant S9 
achieved the greatest accuracies for both analysis tests (94.79\% and 98.33\%), respectively. Participant S15 performed worst for the train - LpOCV target vs. non target analysis but still achieved an accuracy of $72.92 \%$ while participant S12 performed worst overall for the train validation 5-class analysis achieving an accuracy of $68.33 \%$. Overall, participants performed well during the calibration lap with the mean accuracy across all participants for both calibration data tests at $85.17 \%$ and $80.89 \%$, respectively.

TABLE 1: OFFLINE ACCURACY (\%) FOR ALL FIFTEEN PARTICIPANTS OBTAINED DURING THE CALIBRATION LAP.

\begin{tabular}{|c|c|c|}
\hline \multirow{2}{*}{} & \multicolumn{2}{|c|}{ Accuracy (\%) } \\
\cline { 2 - 3 } & LpOCV & Validation \\
\hline Participant No. & 2-class & 5-class \\
\hline S1 & 84.58 & 76.67 \\
\hline S2 & 94.58 & 96.67 \\
\hline S3 & 84.79 & 81.67 \\
\hline S4 & 83.54 & 70.00 \\
\hline S5 & 86.46 & 85.00 \\
\hline S6 & 77.29 & 76.67 \\
\hline S7 & 88.96 & 85.00 \\
\hline S8 & 91.88 & 81.67 \\
\hline S9 & 94.79 & 98.33 \\
\hline S10 & 78.75 & 71.67 \\
\hline S11 & 90.42 & 91.67 \\
\hline S12 & 77.50 & $\mathbf{6 8 . 3 3}$ \\
\hline S13 & 88.75 & 85.00 \\
\hline S14 & 82.29 & 75.00 \\
\hline S15 & $\mathbf{7 2 . 9 2}$ & 70.00 \\
\hline Mean & $\mathbf{8 5 . 1 7}$ & $\mathbf{8 0 . 8 9}$ \\
\hline
\end{tabular}

\subsection{Online Game Control}

Online results are reported and represent the ability of the participant to select the correct stimuli based on the directed cues (cascading arrows on the road surface). Accuracies of up to a maximum of $100 \%$ are reported. For each of the twenty checkpoints available in the racing circuit, if the user selects the correct lane, $5 \%$ of the total achievable accuracy is gained $(5 \% \times 20$ checkpoints $=100 \%$ accuracy). Three laps of the racing circuit were completed by each participant (except S12) and an accuracy is obtained for each completed circuit.

Figures 11 and 12 depict the accuracy and ITR results, respectively. Participants S2, S14 and S8 all achieved $95 \%$ accuracy and ITR of $23 \mathrm{bpm}$, the highest accuracies and ITR for laps 1, 2 and 3, respectively. Participant S15 performed worst across all subjects for all three laps: $45 \%$ (3bpm), 35\% (1bpm) and 35\% (1bpm), for laps 1, 2 and 3, respectively. The mean accuracies and ITR across all participants were $72 \%$ (12bpm), 67\% (10bpm), and $65 \%(10 \mathrm{bpm})$ for laps 1,2 and 3 , respectively. This result shows a clear linear decline in accuracy and ITR as the session progresses from lap to lap (Fig. 13). To verify significance of the difference in performance between laps across all participants, a Wilcoxon signed-rank test was applied. The differences between all lap performances are significant $(p<0.05)$.

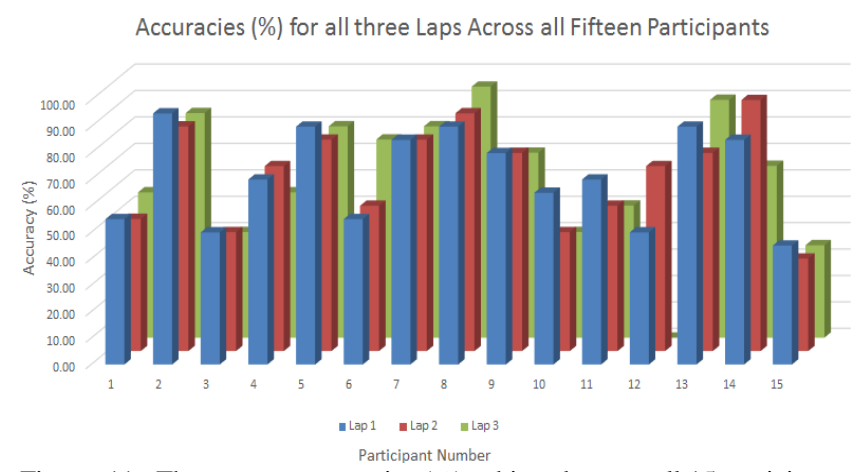

Figure. 11. The average accuracies $(\%)$ achieved across all 15 participants for all three laps.

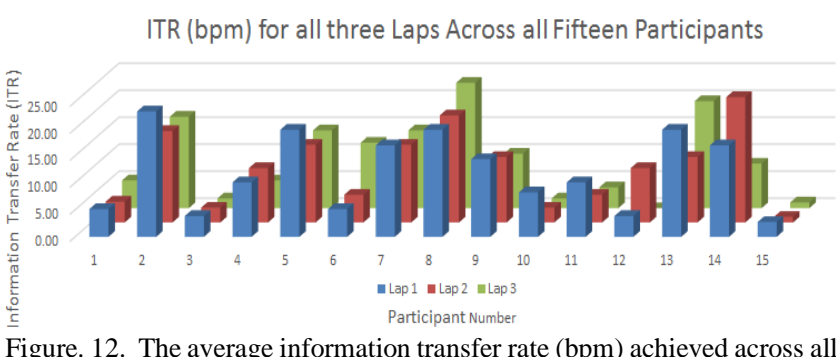
15 participants for all three laps.

Average Accuracies and Player Score Across all Participants for all 3 Laps



Figure. 13. The average accuracies and player scores achieved during the three online laps (see section 2.3 for evaluation of scoring system).

As an additional measure of the performance of each participant and to include additional feedback within the online sessions, a player score was added and displayed to the player during gameplay (the maximum possible score is 10000 points). These are reported in Fig. 13.

The highest scores across all participants for lap 1 was achieved by participant S2 (9600), the highest score for lap 2 was jointly achieved by participants S8 and S14 (9600) and for lap 3, the highest score was achieved by participant S8 (9600). The worst score achieved for lap 1 was obtained by participant S3 who achieved 6400 points and for laps 2 and 3, the worst score was obtained by participant S15 achieving 5600 and 6200 points, respectively. Mean scores were calculated across all subjects and for laps 1, 2 and 3 scores of 8280, 7973 and 7943 were obtained, respectively. As with the online accuracy metric, as the session progresses, the score declined (Fig. 13).

\subsection{Highest-ranking Channels}

The cross-validation test explained in section 3.2 produced the three highest-ranking EEG electrode channels based on the results of LpOCV calculations. We calculated the three most commonly used channels for each participant and these results are presented in Table 2. Table 3 depicts a 
list of the channels used and the corresponding number of times each were used across all participants.

TABLE 2: THE THREE HIGHEST-RANKING CHANNELS FOR EACH PARTICIPANT BASED ON THE LPOCV ANALYSIS.

\begin{tabular}{|c|c|}
\hline Participant No. & Best 3 Channels \\
\hline S1 & P8, Cz, O1 \\
\hline S2 & O1, P7, Oz \\
\hline S3 & O2, P4, Cz \\
\hline S4 & O2, P3, P7 \\
\hline S5 & P7, P3, Cz \\
\hline S6 & P7, Cz, TP7 \\
\hline S7 & O1, P7, P8 \\
\hline S8 & P3, P7, Cz \\
\hline S9 & P7, O1, Cz \\
\hline S10 & $\mathrm{Cz}, \mathrm{O} 1, \mathrm{CP}$ \\
\hline S11 & O2, P8, TP8 \\
\hline S12 & C2, O2, P8 \\
\hline S13 & P7, O1, Cz \\
\hline S14 & O1, P3, P7 \\
\hline S15 & P7, Cz, P3 \\
\hline
\end{tabular}

TABLE 3: THE TWELVE EEG CHANNELS USED AND THE NUMBER OF TIMES EACH WERE CHOSEN ACCORDING TO THE LPOCV ANALYSIS AND ACROSS ALL PARTICIPANTS

\begin{tabular}{|c|c|}
\hline Channel Name & No. of Selections \\
\hline Cz & 10 \\
\hline TP7 & 1 \\
\hline CPz & 1 \\
\hline TP8 & 1 \\
\hline P7 & 10 \\
\hline P3 & 5 \\
\hline Pz & 0 \\
\hline P4 & 1 \\
\hline P8 & 4 \\
\hline O1 & 7 \\
\hline Oz & 1 \\
\hline O2 & 4 \\
\hline
\end{tabular}

In descending order, the channels $\mathrm{Cz}, \mathrm{P} 7$ and $\mathrm{O} 1$ represented the three most commonly used channels across all participants. This is depicted in the topographic plot in fig. 14.

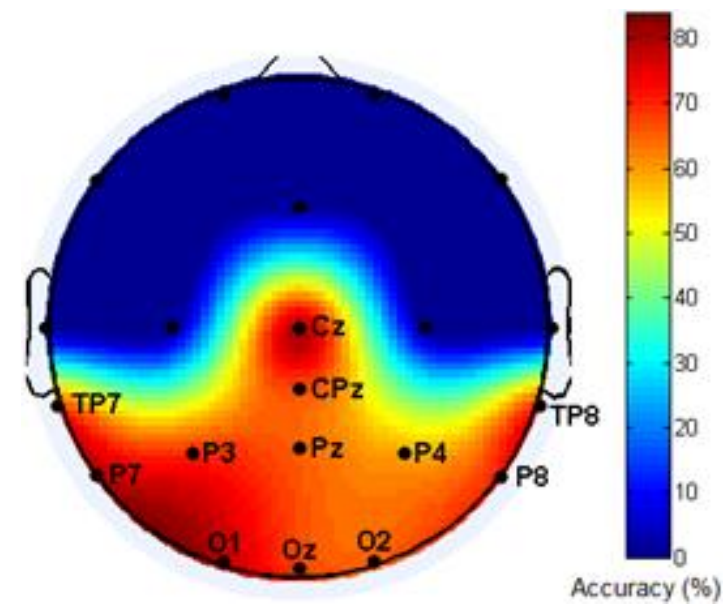

Figure. 14. Topographic illustration depicting the locations of the brain areas that provide the maximum $\mathrm{mVEP}$ discrimination accuracy.

\section{Discussion}

The performance of teenage participants' control over a real-time online 3D car racing game controlled using mVEPs was evaluated in this investigation. For the first time, the study showed mVEP linked to a video-game involving car control. Averaged results across all participants were encouraging and provided evidence for the first time that participants of the 13 to 16 years age group could reliably control the custom-made BCI car racing game with an average accuracy of $72 \%(12 \mathrm{bpm})$ in the first lap, $67 \%(10 \mathrm{bpm})$ in the second lap and $65 \%(10 \mathrm{bpm})$ in the third lap. The results varied significantly across subjects, which is normal for first session BCI experiments with limited calibration trials, where there are number of confounding factors that can impact on performance, including excitability and anxiety in relation to taking part in experiments as well as cognitive load and challenge during familiarisation with the paradigm. Nevertheless, accuracies as high as $95 \%(23 \mathrm{bpm})$ were recorded for three of the participants. The average accuracy across the seven top performing participants is $80 \%(14.4 \mathrm{bpm})$ while the average across the eight worst performing participants is $57 \%$ (5.7bpm). We have found that as the session progressed, BCI accuracies linearly decreased. The degradation in accuracy as the session progressed may relate to subject fatigue, reduction in interest or waning concentration. Participants may have found monotony in the lack of variation after each consecutive lap played and the slow pace of the games, compared to normal games that may have had an impact on motivation. Of course, setup issues could also be a cause of the decline in accuracy e.g., the EEG cap loosening over time or electrode gels drying or dispersing. In certain cases, participants performed better during later laps. This may be as a result of improved understanding of the control and gameplay strategy and less distraction by the games environment as the participants habituated to the graphics and distraction in the game.

Our findings show that when using leftwards motion of the stimuli, the three most commonly used EEG channels $(\mathrm{Cz}, \mathrm{P} 7$ and $\mathrm{O} 1)$ are located on the left visual hemisphere. This finding is consistent with previous studies where leftwards motion of the stimuli was also used [37][36][39][40][30]. These locations also correspond to the middle temporal (MT) and medial superior temporal (MST) areas of the brain which is specialised for the processing of motion. The asymmetrical effect of the mVEP features also supports the right/ left visual field asymmetry effect on contralateral hemisphere findings described in [52].

As the field of neuro-gaming has gained traction, particularly over the past number of years [12][13], it is important that professional video game design for neurogaming is at a high standard. Most previous BCI video games have traditionally offered only basic interfaces, graphics or gameplay elements and, although this has helped to progress the field, future studies should recognise that basic game elements such as those seen in most previous video-games tested within BCI frameworks may not be appealing to the wider gaming population. Therefore, to ensure that BCI-controlled video-games of the future offer engaging, attractive and appealing graphics and gameplay elements, work still needs to be done to study the effects of 
these properties within BCI games. It is important to note, however, that basic graphics and gameplay do not necessarily make for an unappealing game, in fact, some of the most successful video-games of all time have employed basic graphics and gameplay namely Pong [53], Tetris [54] and Pacman [55]. To appeal to the wider gaming population, complex graphics and gameplay within BCI video-games is still an important and understudied topic. If BCGI is to become a viable entertainment medium in the future, careful consideration must be given to the current state-of-the-art in video-gaming.

For this study, fifteen teenage participants were selected while they attended a summer school. This is a relatively understudied demographic group for BCI studies. For the future progress of BCI technology, it is important that $\mathrm{BCI}$ performance of users of all ages be investigated.

\section{Limitations}

A number of limitations were observed during the course of the current study. In order to gather enough data to enable reliable classification, we employed five trials per checkpoint and averaged responses were fed into the classifier for classification. Therefore, a five second time period was required to gather the input from the participants between checkpoints. Aside from this, to allow suitable preparation time prior to the commencement of the mVEP stimuli, activation of the stimuli did not begin until three seconds after being presented on screen. These time restrictions dictated the slow speed of the in-game car. Some participants did not like the slow-paced gameplay compared to that of commercially available video-games. This is being addressed in a later study where faster car speed may be possible using less repetitions of the stimuli to make a decision about which stimulus the user is attending.

As the participants were recruited during a summer school, there were time limitations placed on the time for each participant's session. Participants were instructed on the tasks required for both the offline and online games before their session and some may not have fully understood the task. This limitation may be addressed in later studies, by introducing longer pre-session instruction periods and conducting a multisession evaluation of performance for larger study cohort. As the summer school offers a period of densely packed training to the students, better results may have been achieved during a more relaxed period during the year.

We decided to select a maximum of three electrode channels over the most reactive brain areas to form feature vectors, as this normally provides reasonable accuracy. Additionally, the feature extraction approach focused on a standardised method and only the $100-500 \mathrm{~ms}$ post stimulus period was used. Subject-specific selection of this window position and width along with selection of the maximum number of channels from which to create a feature and optimising parameters of the feature extraction would have increased performance however, as the session for each participant was time-limited, and we were collecting calibration data, calibrating algorithms for each participant and providing online feedback on three laps in single session, extensive subject-specific calibration was not feasible.

This study used the Unity 3D games engine to present the stimuli and games and send markers/ triggers to a Matlab session-based interface, over a locally connected network using the UDP protocol. Each element of this framework has inherent limitations and variations, resulting in the simultaneous registration of the stimulus and EEG response being compromised e.g., time delays and discrepancies can cause jitter in the triggering of the stimulus onset on the $\mathrm{mVEP}$ in the EEG recording which can impact on detection accuracy. There is no doubt that a more exact real-time processing framework, for example, and Matlab Simulink and dedicated triggering software and hardware would have increased the accuracy of the system. However, an aim of the study was to validate a framework that used commercially orientated technologies and protocols.

\section{Conclusion}

Our findings demonstrate the feasibility of a reliable, online, low visually fatiguing paradigm which can be employed in future BCI video-games with commercialgrade graphics, gameplay elements and technologies. We have found that the commercial-grade graphics used within the $3 \mathrm{D}$ racing game environment were not detrimental to mVEP discrimination and acceptable control was achieved in a single session by BCI naïve, teenage participants. There is likely to be other factors associated with engaging teenagers in an experiment and maintaining interest, attention and motivation. Games are likely to alleviate any limitation in these processes during BCI studies, however, the results presented here show that as the session progressed and across each successive lap of the racing circuit played, a decline in performance was observed. This may have been caused by the use of the same game level/ track and lack of variation in speed or pace of the games throughout the session, as well as the differential in speed of the games compared to commercial games. Improving this part of the experiment is the topic of ongoing research where three different levels of gameplay in the form of three cars each using a gradually increasing speed are being investigated. The speed of the car during these levels will dictate the time available to classify the intended target derived from the mVEP responses. A lower number of trials used to make a decision will increase the speed of the decisions by the BCI but may also have an impact on accuracy. We aim to compare the performance of children and adults and robustness of performance across multiple sessions.

\section{References}

[1] J. R. Wolpaw, N. Birbaumer, D. J. McFarland, G. Pfurtscheller, and T. M. Vaughan, "Brain-computer interfaces for communication and control.," Clin. Neurophysiol., vol. 113, no. 6, pp. 767-91, Jun. 2002.

[2] L. a Farwell and E. Donchin, "Talking off the top of your head: toward a mental prosthesis utilizing event-related brain potentials.," Electroencephalogr. Clin. Neurophysiol., vol. 70, no. 6, pp. 510-23, Dec. 1988.

[3] B. Hong, F. Guo, T. Liu, X. Gao, and S. Gao, "N200-speller using motion-onset visual response.," Clin. Neurophysiol., vol. 120, no. 9, pp. 1658-66, Sep. 2009.

[4] X. Chen, Y. Wang, M. Nakanishi, X. Gao, T. Jung, and S. Gao, "High-speed spelling with a noninvasive brain - computer interface," Procceedings Natl. Acad. Sci. United States Am., vol. 112, no. 44, pp. 1-11, 2015. 

R. Milla, "A brain-actuated wheelchair: Asynchronous and noninvasive Brain - computer interfaces for continuous control of robots," Clin. Neurophysiol., vol. 119, pp. 2159-2169, 2008.

G. R. Müller-Putz and G. Pfurtscheller, "Control of an electrical prosthesis with an SSVEP-based BCI," IEEE Trans. Biomed. Eng., vol. 55, no. 1, pp. 361-364, 2008.

J. J. Daly, J. R. Wolpaw, and L. S. Cleveland, "Brain computer interfaces in neurological rehabilitation," Lancet Neurol., vol. 7, no. 11, pp. 1032-1043, 2008.

G. Pfurtscheller, R. Scherer, and C. Neuper, "Rehabilitation with Brain-Computer Interface Systems," Computer (Long. Beach. Calif)., vol. 41, no. 10, pp. 58-65, 2008. "Sparse Bayesian Classification of EEG for Brain - Computer Interface," IEEE Trans. Neural Networks Learn. Syst., pp. 1-12, 2015.

[10] Y. U. Zhang, G. Zhou, J. Jin, Q. Zhao, X. Wang, and A Cichocki, "Aggregation of Sparse Linear Discriminant Analyses for Event-related Potential Classification in Brain-computer Interface," Int. J. Neural Syst., vol. 24, no. 1, pp. 1-15, 2014.

[11] F. Lotte and M. Congedo, "A review of classification algorithm for EEG-based brain - computer interfaces," J Neural Eng, vol. 4, pp. 1-13, 2007.

[12] D. Marshall, D. Coyle, S. Member, S. Wilson, and M. Callaghan, "Games, Gameplay, and BCI : The State of the Art," IEEE Trans. Comput. Intell. AI Games, vol. 5, no. 2, pp. 82-99, 2013.

[13] A. Nijholt, D. P.-O. Bos, and B. Reuderink, "Turning shortcomings into challenges: Brain-computer interfaces for games," Entertain. Comput., vol. 1, no. 2, pp. 85-94, Apr. 2009.

[14] D. Coyle, J. Principe, F. Lotte, and A. Nijholt, "Guest Editorial: Brain/neuronal - Computer game interfaces and interaction," IEEE Trans. Comput. Intell. AI Games, vol. 5, no. 2, pp. 77-81, Jun. 2013

[15] newzoo GAMES, “2016 Global Games Market Report," Amsterdam, June 2016.

[16] J. Jin, B. Z. Allison, Y. Zhang, X. Wang, and A. Cichocki, “An ERP-based BCI using an Oddball Paradigm with Different Faces and Reduced Errors in Critical Functions," Int. J. Neural Syst., vol. 24, no. 8, pp. 1-14, 2014.

[17] E. Yin, T. Zeyl, R. Saab, T. Chau, D. Hu, and Z. Zhou, "A Hybrid Brain - Computer Interface Based on the Fusion of P300 and SSVEP Scores," IEEE Trans. Neural Syst. Rehabil. Eng., vol. 23, no. 4, pp. 693-701, 2015.

[18] J. Jin, B. Z. Allison, E. W. Sellers, C. Brunner, P. Horki, X. Wang, and C. Neuper, "An adaptive P300-based control system,” J. Neural Eng., vol. 8, pp. 1-14, 2011.

[19] J. Jin, E. W. Sellers, S. Zhou, Y. Zhang, X. Wang, and A Cichocki, "A P300 Brain - Computer Interface Based on a Modification of the Mismatch Negativity Paradigm," Int. J. Neural Syst., vol. 25, no. 3, pp. 1-12, 2015.
[21] A. Finke, A. Lenhardt, and H. Ritter, "The MindGame: a P300based brain-computer interface game.," Neural Netw., vol. 22, no. 9, pp. 1329-33, Nov. 2009.

[22] L. Korczowski, M. Congedo, C. Jutten, L. Korczowski, M. Congedo, C. Jutten, S. Classification, L. Korczowski, M. Congedo, and C. Jutten, "Single-Trial Classification of MultiUser P300-Based Brain-Computer Interface Using Riemannian Geometry," in 37TH Annual International Conference of the IEEE Engineering in Medicine and Biology Society (EMBC), 2015, pp. 1769-1772.

[23] E. Yin, Z. Zhou, J. Jiang, F. Chen, Y. Liu, and D. Hu, "A speedy Hybrid BCI spelling approach combining P300 and SSVEP.," IEEE Trans. Biomed. Eng., vol. 61, no. 2, pp. 473-83, Feb. 2014.

[24] E. Yin, Z. Zhou, J. Jiang, Y. Yu, and D. Hu, "A Dynamically Optimized SSVEP Brain - Computer Interface (BCI) Speller," IEEE Trans. Biomed. Eng., vol. 62, no. 6, pp. 1447-1456, 2015.

[25] P. Martinez, H. Bakardjian, and A. Cichocki, "Fully online multicommand brain-computer interface with visual neurofeedback using SSVEP paradigm.," Comput. Intell. Neurosci., vol. 2007, pp. 1-9, Jan. 2007.

[26] E. C. Lalor, S. P. Kelly, C. Finucane, R. Burke, R. Smith, R. B. Reilly, and G. McDarby, "Steady-State VEP-Based BrainComputer Interface Control in an Immersive 3D Gaming Environment," EURASIP J. Adv. Signal Process., vol. 2005, no. 19, pp. 3156-3164, 2005.

[27] G. Bin, X. Gao, Y. Wang, Y. Li, B. Hong, and S. Gao, "A highspeed BCI based on code modulation VEP," J Neural Eng, vol. 8, pp. 1-5, 2011.

[28] M. Spuler, “A Brain-Computer Interface (BCI) system to use arbitrary Windows applications by directly controlling mouse and keyboard," Proc. Annu. Int. Conf. IEEE Eng. Med. Biol. Soc. EMBS, vol. 2015-Novem, pp. 1087-1090, 2015.

[29] H. Riechmann, A. Finke, and H. Ritter, "Using a cVEP-Based Brain-Computer Interface to Control a Virtual Agent," IEEE Trans. Neural Syst. Rehabil. Eng., vol. 24, no. 6, pp. 692-699, 2016.

[30] F. Guo, B. Hong, X. Gao, and S. Gao, "A brain-computer interface using motion-onset visual evoked potential.," J. Neural Eng., vol. 5, no. 4, pp. 477-85, Dec. 2008.

[31] W. T. Newsome, R. H. Wurtz, and H. Komatsu, "Relation of Cortical Areas MT and MST to Pursuit Eye Movements. II. Differentiation of Retinal from Extraretinal Inputs," $J$. Neurophysiol., vol. 60, no. 2, pp. 604-620, 1988.

U. J. Ilg, "The role of areas MT and MST in coding of visual motion underlying the execution of smooth pursuit," Vision Res., vol. 48, no. 20, pp. 2062-2069, 2008.

[33] Z. Kubová, M. Kuba, H. Spekreijse, and C. Blakemore, "Contrast dependence of motion-onset and pattern-reversal evoked potentials.," Vision Res., vol. 35, no. 2, pp. 197-205, Jan. 1995.

[34] F. Di Russo, M. I. Sereno, S. Pitzalis, and S. A. Hillyard, "Cortical Sources of the Early Components of the Visual Evoked Potential,” vol. 111, pp. 95-111, 2001.

[35] M. Kuba and Z. Kubova, "Visual Evoked Potentials Specific for Motion onset," Doc. Ophthalmol., vol. 80, pp. 83-89, 1992. 
[36] D. Marshall, S. Wilson, and D. Coyle, "Motion-Onset Visual Evoked Potentials for Gaming," in 8th International Conference on Computer Games, Multimedia and Allied Technology, 2015, pp. 155-164.

[37] D. Marshall, R. Beveridge, S. Wilson, and D. Coyle, "Interacting with Multiple Game Genres using Motion Onset Visual Evoked Potentials," in The 20th International Conference on Computer Games, 2015, pp. 18-27.

[38] W. Li, M. Li, and J. Zhao, "Control of humanoid robot via motion-onset visual evoked potentials," Front. Syst. Neurosci., vol. 8, no. January, pp. 1-11, 2015.

[39] R. Beveridge, D. Coyle, and S. Wilson, "3D Game Graphic Complexity Effects on Motion-Onset Visual Evoked Potentials," in 8th Annual International Conference on Computer Games Multimedia and Allied Technologies, 2015, pp. 139-147.

[40] R. Beveridge, D. Marshall, S. Wilson, and D. Coyle, "Classification Effects on Motion-Onset Visual Evoked Potentials using Commercially Available Video Games," in The 20th International Conference on Computer Games, 2015, pp. 28-37.

[41] Sony Computer Entertainment, "Gran Turismo," 2016. [Online]. Available: http://www.gran-turismo.com/gb/. [Accessed: 05Aug-2016].

[42] Sony Interactive Entertainment, "Playstation," 2016. [Online] Available: https://www.playstation.com/en-gb/. [Accessed: 05Aug-2016].

[43] Sony Computer Entertainment, "Crash Bandicoot," 2016. [Online]. Available: https://www.playstation.com/engb/games/crash-bandicoot-ps3/. [Accessed: 05-Aug-2016].

[44] R. Beveridge, D. Coyle, and S. Wilson, "Virtual Reality, Graphics and mVEP Classification," in Proceedings of the 6th International Brain-computer Interface Meeting Organised by the BCI Society, 2016, p. 223.

[45] Oculus, "Oculus Rift,” 2016. [Online]. Available: http://www.oculus.com/. [Accessed: 05-Aug-2016].

[46] Guger Technologies, "g.tec Medical Engineering," 2016. [Online]. Available: http://www.gtec.at/. [Accessed: 05-Aug2016].

[47] NI, "National Instruments," 2016. [Online]. Available: http://www.ni.com/en-gb.html. [Accessed: 13-Oct-2016].

[48] MathWorks, "MATLAB," 2016. [Online]. Available: http://www.mathworks.co.uk/products/matlab/. [Accessed: 05Aug-2016].

[49] Unity Technologies, "Unity 3D," 2016. [Online]. Available: http://unity3d.com/. [Accessed: 05-Aug-2016].

[50] J. R. Wolpaw, N. Birbaumer, W. J. Heetderks, D. J. McFarland, P. H. Peckham, G. Schalk, E. Donchin, L. a Quatrano, C. J. Robinson, and T. M. Vaughan, "Brain-computer interface technology: a review of the first international meeting.," IEEE Trans. Rehabil. Eng., vol. 8, no. 2, pp. 164-173, 2000.

\section{O. Doherty, Y. K. Meena, H. Raza, H. Cecotti, and G.} Prasad, "Exploring Gaze-Motor Imagery Hybrid BrainComputer Interface design," in IEEE International Conference on Bioinformatics and Biomedicine, 2014, pp. 335-339.
[52] S. a. Heinze, H. J., Mangun, G. R., Burchert, W., Hinrichs, H., Scholz, M. Münte, T. F., Gös, A., Scherg, M., Johannes, S., Hungeshagen, H., Gazzinga, M. S. \& Hillyard, "Combined Spatial and Temporal Imaging of Brain Activity During Visual Selective Attention in Humans," Nature, vol. 372. pp. 543-546, 1994.

[53] R. Baer, "Pong." 1972.

[54] A. Pajitnov, “Tetris.” Tetris Holding, 1984.

[55] "PACMAN." Namco, 1980. 
